\title{
Pathologic response of ductal carcinoma in situ to neoadjuvant systemic treatment in HER2-positive breast cancer
}

\author{
Emma J. Groen ${ }^{1,2} \cdot$ Marieke E. M. van der Noordaa $^{3} \cdot$ Michael Schaapveld $^{4} \cdot$ Gabe S. Sonke $^{5} \cdot$ Ritse M. Mann ${ }^{6,7}$. \\ Mette S. van Ramshorst ${ }^{8}$ - Esther H. Lips ${ }^{2}$ - Marie-Jeanne T. F. D. Vrancken Peeters ${ }^{3}$. Frederieke H. van Duijnhoven ${ }^{3}$. \\ Jelle Wesseling ${ }^{1,2,9}$
}

Received: 20 November 2020 / Accepted: 17 April 2021 / Published online: 4 May 2021

(c) The Author(s) 2021

\begin{abstract}
Purpose The presence of extensive ductal carcinoma in situ (DCIS) adjacent to HER2-positive invasive breast cancer (IBC) is often a contra-indication for breast-conserving surgery, even in case of excellent treatment response of the invasive component. Data on the response of DCIS to neoadjuvant systemic treatment (NST) are limited. Therefore, we estimated the response of adjacent DCIS to NST-containing HER2-blockade in HER2-positive breast cancer patients and assessed the association of clinicopathological and radiological factors with response.

Methods Pre-NST biopsies were examined to determine presence of DCIS in all women with HER2-positive IBC treated with trastuzumab-containing NST \pm pertuzumab between 2004 and 2017 in a comprehensive cancer center. When present, multiple DCIS factors, including grade, calcifications, necrosis, hormone receptor, and Ki-67 expression, were scored. Associations of clinicopathological and radiological factors with complete response were assessed using logistic regression models.

Results Adjacent DCIS, observed in 138/316 patients with HER2-positive IBC, was eradicated after NST in 46\% of patients. Absence of calcifications suspicious for malignancy on pre-NST mammography (odds ratio (OR) 3.75; 95\% confidence interval (95\% CI) 1.72-8.17), treatment with dual HER2-blockade (OR 2.36; 95\% CI 1.17-4.75), a (near) complete response on MRI (OR 3.55; 95\% CI 1.31-9.64), and absence of calcifications (OR 3.19; 95\% CI 1.34-7.60) and Ki-67>20\% in DCIS (OR 2.74; 95\% CI 1.09-6.89) on pre-NST biopsy were significantly associated with DCIS response.

Conclusions As DCIS can respond to NST containing HER2-blockade, the presence of extensive DCIS in HER2-positive breast cancer before NST should not always indicate a mastectomy. The predictive factors we found could be helpful when considering breast-conserving surgery in these patients.
\end{abstract}

Keywords HER2-positive breast cancer · Ductal carcinoma in situ $\cdot$ Response $\cdot$ Neoadjuvant systemic treatment

\section{Abbreviations}

NST Neoadjuvant systemic therapy

pCR Pathologic complete response

HER2 Human epidermal growth factor receptor 2

IBC Invasive breast cancer

HR Hormone receptor

Emma J. Groen and Marieke E. M. van der Noordaa shared first authors.

Frederieke H. van Duijnhoven and Jelle Wesseling shared last authors.

Jelle Wesseling

j.wesseling@nki.nl

Extended author information available on the last page of the article

$\begin{array}{ll}\text { BCS } & \text { Breast-conserving surgery } \\ \text { DCIS } & \text { Ductal carcinoma in situ } \\ \text { NKI } & \text { Netherlands Cancer Institute } \\ \text { ER } & \text { Estrogen receptor } \\ \text { PR } & \text { Progesterone receptor } \\ \text { OR } & \text { Odds ratio } \\ \text { 95\% CI } & 95 \% \text { Confidence interval }\end{array}$

\section{Background}

Neoadjuvant systemic therapy (NST) that contains trastuzumab in addition to neoadjuvant chemotherapy leads to high pathologic complete response (pCR) rates in patients with human epidermal growth factor receptor 2 
(HER2)-positive invasive breast cancer (IBC) [1-3]. Even higher pCR rates are seen when a trastuzumab-containing regimen is combined with the HER2-targeted antibody pertuzumab (i.e., dual HER2-blockade), with pCR rates of up to $80 \%$ reported in the HER2-positive/hormone receptor (HR)-negative subtype [4-9]. These excellent response rates allow for frequent conversion from mastectomy to breastconserving surgery (BCS).

The presence of ductal carcinoma in situ (DCIS) adjacent to IBC, observed in 57-72\% of HER2-positive breast cancer patients, may, however, impede this de-escalation of surgery, as DCIS is considered insensitive to systemic treatment [10-17]. A lower proliferative state, more intact physiological resistance mechanisms compared to IBC and a less receptive microenvironment to chemotherapeutic agents due to a protective basal membrane and less dense microvasculature have been put forward as potential causes for this therapy resistance [18-20]. Therefore the presence of a large area of calcifications on mammography or non-mass enhancement on MRI, both of which may be associated with DCIS, and/or extensive DCIS adjacent to IBC in pre-NST biopsies are often considered contra-indications for BCS, even in patients with radiological complete response of the tumor on breast MRI [21, 22].

However, data on the response of DCIS to NST are limited. A few studies have shown that DCIS may sometimes respond to NST [14, 23-25]. Two retrospective studies evaluating response of DCIS adjacent to HER2-positive breast cancer found that $36-51 \%$ of these DCIS lesions were eradicated after trastuzumab-containing NST combined with pertuzumab in a small subgroup [24, 25].

It is, however, not possible to predict which DCIS lesions adjacent to HER2-positive IBC will respond to NST. Imaging studies have difficulties to identify residual DCIS after NST, as the extent of calcifications on mammography after NST is very poorly associated with the pathologic response or residual size of invasive or in situ components $[14,16$, $26,27]$. Therefore, performing BCS in patients with extensive DCIS is challenging, even when an excellent treatment response of their IBC has been achieved. To facilitate potential de-escalation of surgery in the future in this patient group, we aim to estimate the response of adjacent DCIS to NST containing HER2-blockade in a large series of HER2positive breast cancer patients and to identify clinicopathological and radiological factors that predict response.

\section{Methods}

\section{Patient and data collection}

All women $\geq 18$ years diagnosed with HER2-positive IBC who received NST containing HER2-blockade at the
Netherlands Cancer Institute (NKI) between January 2004 and November 2017 were selected from the prospectively maintained NKI's tumor registry.

Detailed patient, imaging, tumor and treatment characteristics were extracted from medical records. HER2 and HR status of IBC were assessed in all patients according to the Dutch guidelines. HR status was considered positive when $\geq 10 \%$ of luminal epithelial cells showed nuclear estrogen receptor (ER) expression, irrespective of progesterone receptor (PR) expression [22, 28]. Ki-67 in IBC was categorized into low ( $\leq 20 \%$ of expression) and high ( $>20 \%$ expression) proliferation. Neoadjuvant chemotherapy regimens were categorized into taxanebased, anthracycline plus taxane-based or other. Type of HER2-blockade was registered (i.e., trastuzumab alone or dual HER2-blockade with trastuzumab and pertuzumab). Patients underwent both mammography and MRI pre-NST. All lesions were assessed by radiologists according to the BI-RADS lexicon [29]. For each tumor the size of the largest mass lesion, i.e., the index lesion, was reported as the largest diameter in the axial plane. In addition, the extent of the tumor was reported, being the size of the tumor area including surrounding satellites and non-mass enhancement. The presence and extent of calcifications suspicious for malignancy on pre-NST mammography was noted. A dedicated breast radiologist (RMM) reassessed mammographic images when relevant information regarding the presence or level of suspicion of calcifications (i.e., whether the calcifications were considered benign or suspicious for malignancy) was missing in the original report.

Tumor response was assessed on MRI after completion of NST, since MRI is superior to mammography in determining the presence and size of residual disease, and was categorized into (near) complete versus partial or no radiological response [30]. Radiological complete response was defined as no residual enhancement within the original tumor bed after NST. Near complete response was reported when only minimal residual enhancement (either some foci, or a diffuse glow) was visualized within the original tumor bed, without any components that were clearly identifiable as part of the original tumor. Post-NST mammography was not performed.

For women treated with breast-conserving surgery, the tumor was marked with a clip marker and localized with use of radio-guided occult lesion localization in the earlier years of our study cohort. In some patients, localization of the tumor was done with use of a wire. From 2007 the tumor was typically marked with an iodine seed prior to NST [31]. Breast-conserving surgery was planned using post-NST MRI findings. Specimen radiography was performed for all lumpectomies and for mastectomy specimens if a substantial pre-NST DCIS component was present to guide tissue sampling. 
This study was approved by the institutional review board of the NKI.

\section{Pathology review}

A dedicated breast pathologist (EJG) re-examined all preNST biopsies, blinded for response, to determine whether DCIS was present adjacent to IBC. These pre-NST biopsies mostly targeted the invasive component and were preferentially obtained under ultrasound guidance using a $14 \mathrm{G}$ core biopsy needle. In lesions that were ultrasound occult or presented as mammographic calcifications only, stereotactic biopsy was performed using a $9 \mathrm{G}$ vacuum needle. The number of available tissue cores was documented. If adjacent DCIS was present, the following histopathological DCIS features were scored: number of DCIS ducts, grade $(1,2$ or 3) according to Holland criteria, dominant growth pattern (clinging, (micro-)papillary, cribriform, or solid), presence of calcifications, necrosis, periductal lymphocytic infiltrate, (type of) periductal fibrosis and mitotic activity (see scoring form in Supplementary methods) [32]. When slides originally stained with ER, PR, HER2 and Ki-67 contained DCIS, their expression was scored in the DCIS component (see details on antibodies in Supplementary methods). HER2 and HR status of DCIS were determined similarly as for IBC. As little is known about the distribution of Ki-67 in DCIS, Ki-67 in DCIS was categorized into two categories with the median used as cut-off value: low proliferation when $\leq 20 \%$ of cells showed expression and high proliferation when $>20 \%$ of cells showed expression.

Response of DCIS was defined as complete eradication of DCIS after NST. Data on the presence of residual DCIS in post-NST surgical specimens were retrieved from pathology reports. The number of slides that were originally examined was also noted. When no residual DCIS was described in the reports from women in whom adjacent DCIS was found in pre-NST biopsies, pathology slides were re-examined to affirm the eradication of DCIS.

\section{Statistical analysis}

Descriptive statistics were used for patient, imaging, tumor and treatment characteristics. Included and excluded patients were compared, as were included patients with and without adjacent DCIS on pre-NST biopsy, using Pearson's chisquared test for categorical values and Wilcoxon rank-sum test or $t$-test for continuous variables. Adjacent DCIS was defined as any presence of DCIS on pre-NST biopsy.

Associations of clinicopathological and radiological factors with the response of DCIS to NST were assessed using logistic regression models. A stepwise regression was undertaken using forward selection. Variables were entered in multivariable models, based on a $P$ value $\leq 0.05$ in univariable analyses with elimination of variables at a threshold $P$ value of $>0.05$ in the multivariable analysis. Missing data on these eligible variables were imputed using chained equations (MICE) creating 50 datasets. Frequency of missingness was $1 \%$ for suspicious calcifications on mammography, $5 \%$ for tumor response on MRI, $5 \%$ for calcifications in DCIS in the biopsy, and 44\% for Ki-67 expression in DCIS. Estimates from the imputed data sets were pooled using Rubin's rule [33]. All tests were two-sided and $P$ values $\leq 0.05$ were considered statistically significant. All statistical analyses were performed using Stata/SE (version 13.1, Statacorp).

\section{Results}

During the inclusion period, 489 patients with HER2positive IBC received NST containing HER2-blockade at the Netherlands Cancer Institute. After exclusion of 173 patients, mainly because their pre-NST biopsies were not available for review (76\%), 316 patients were available for further analyses (see flow diagram for patient selection and exclusions in Fig. 1). Included patients more often had lower-stage disease and were more frequently treated by a taxane-only regimen than excluded patients (see Supplementary Table 1, demonstrating clinicopathological characteristics of included and excluded patients).

Adjacent DCIS was observed in pre-NST biopsies from 138 out of 316 patients (44\%). In 63 patients (20\%) multiple biopsies were taken; in ten of these patients these biopsies targeted an area of calcifications or non-mass enhancement suspicious for an adjacent DCIS component. The remainder was targeted at the IBC only. Presence of adjacent DCIS increased with the number of examined tissue cores $(P=0.001)$, decreased with age $(P=0.047)$, was more frequent when suspicious calcifications were present on mammography $(P=0.005)$ and, in those with suspicious calcifications, increased when the extent of calcifications on the mammography was larger $(P=0.022$; Table 1$)$. Although patients with adjacent DCIS more often had a lower grade (grade $1+2$ versus grade 3 ) of IBC, this association did not reach statistical significance $(P=0.054)$. At histopathological re-examination of pre-NST biopsies, DCIS was assigned grade 1 in $2 \%$ of patients, grade 2 in $45 \%$ and grade 3 in $53 \%$. The HER2 status of DCIS could be assessed in $86 / 138$ patients and was positive in $92 \%$, equivocal in $7 \%$ (in these patients no SISH was available) and negative in $1 \%$ of patients. HR status of DCIS was positive in $63.5 \%$ and negative in $36.5 \%$ out of the 85 patients for whom HR stains were available. In $82 \%$ of these 85 patients, HR status of DCIS and IBC was concordant. In case of discordancy, a combination of HR-positive DCIS adjacent to HR-negative IBC was most frequently observed. In 9 out of 34 patients with 
Fig. 1 Flow diagram for patient selection and exclusions. $I B C$ invasive breast cancer; $T z t$ trastuzumab; NST neoadjuvant systemic therapy; $n$ number; $i B C$ ipsilateral breast cancer. ${ }^{\text {a }}$ In situ and invasive breast cancer. ${ }^{\mathrm{b}}$ Second primary malignancies, for which treatment may interfere with response evaluation of DCIS to NST

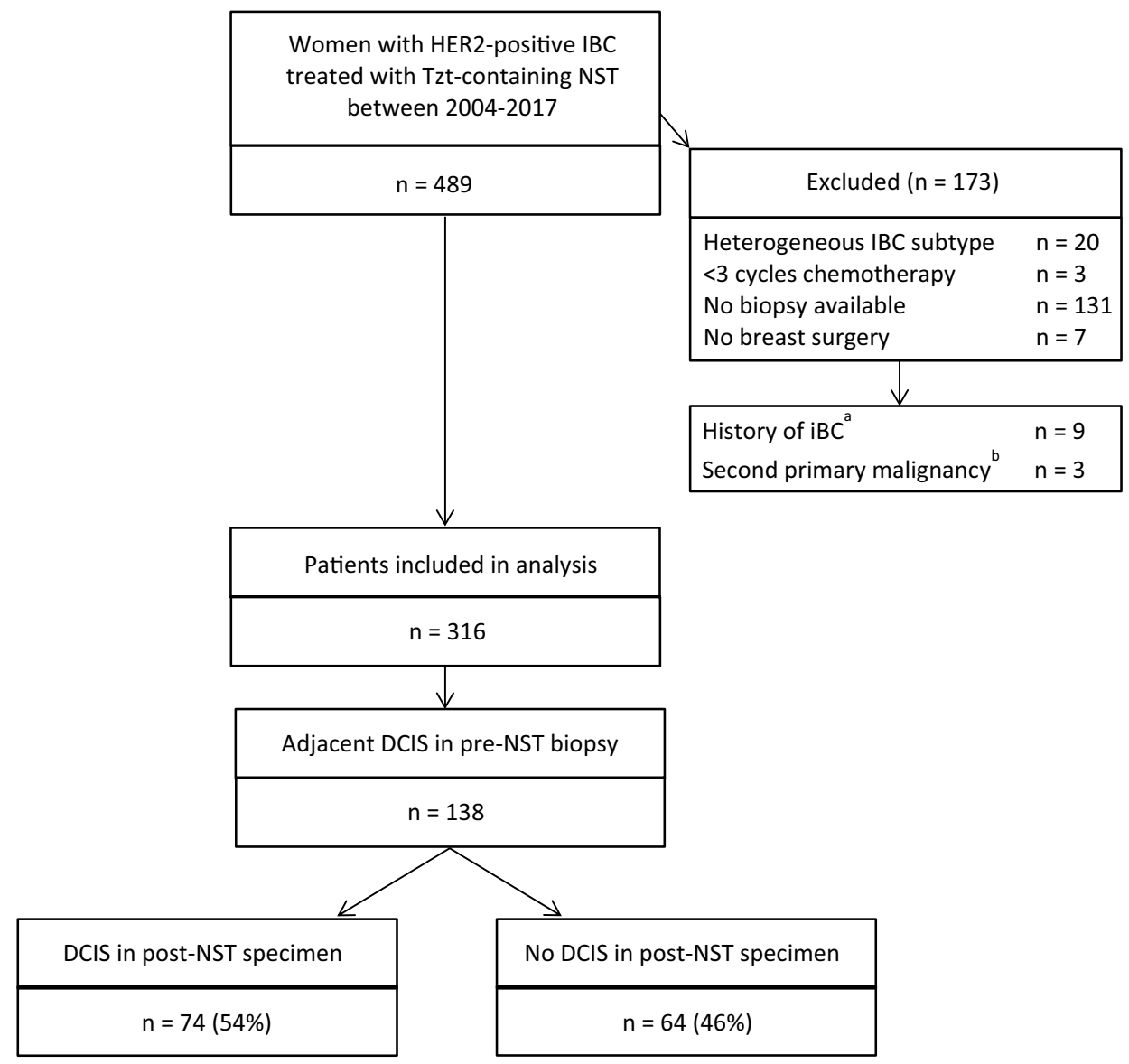

HR-negative IBC the DCIS component was HR positive (26\%), of these patients $67 \%$ showed a complete response (6/9 patients). Conversely, in the 6 (12\%) out of 51 patients with HR-positive IBC with adjacent HR-negative DCIS, the response rate was $50 \%$.

Of the 138 patients with adjacent DCIS on pre-NST biopsy, $80 \%$ were treated with a taxane-based regime, $19 \%$ with an anthracycline plus taxane-based regime and in $1 \%$ with another regime. Sixty-one percent of patients received trastuzumab and 39\% received dual HER2-blockade with trastuzumab and pertuzumab. A (near) complete radiological response on MRI was observed in $82 \%$ of patients. Seventyseven patients were initially treated by lumpectomy and 61 by mastectomy. Resection margins were free in $87 \%$ of the women treated by breast-conserving surgery (67/77). Margins were involved in 10 patients due to irradically removed DCIS $(n=6)$, IBC $(n=1)$ or both $(n=3)$. Re-surgery was performed in 6 patients (re-lumpectomy in 2 and mastectomy in 4 patients) leading to a final free margin status. In the remaining 4 patients, who all showed only focally involved margins, no re-surgery was performed.

The median number of slides examined from post-NST surgical specimens for women with adjacent DCIS was 10 (interquartile range 8-14). After NST, DCIS was eradicated in 64 out of 138 patients (46\%). The number of examined slides did not differ between patients with or without residual DCIS $(P=0.20)$. In $59 \%$ of patients who showed DCIS response, breast-conserving surgery was performed (without considering other pre-NST factors), while in the nonresponder group this was $47 \%(P=0.16)$. In women with residual DCIS after NST, DCIS was found without IBC in 39/74 women (53\%; Table 2). In contrast, in women with residual IBC, IBC without DCIS was found only in 9 out of 44 patients (20\%). Among the 178 patients in whom adjacent DCIS was not found on pre-NST biopsy, 61 patients (34\%) had DCIS after NST based on pathology reports, which was associated with residual IBC in 38 patients (62\%).

\section{Association between clinicopathological and radiological factors and response of DCIS to NST}

The clinico-radiological factors, absence of suspicious calcifications on mammography (odds ratio (OR) 3.75; 95\% confidence interval (95\% CI) 1.72-8.17), treatment with dual HER2-blockade (OR 2.36; 95\% CI 1.17-4.75) and a (near) complete response on MRI (OR 3.55; 95\% CI 1.31-9.64) were associated with DCIS response in univariable analysis (Tables 3,4), as were the histopathological factors absence 
Table 1 Clinico-radiological and IBC factors in patients with and without adjacent DCIS

\begin{tabular}{|c|c|c|c|}
\hline Factors & $\begin{array}{l}\text { DCIS } n(\%)^{\mathrm{a}} \\
n=138(43.7)\end{array}$ & $\begin{array}{l}\text { No DCIS } n(\%) \\
n=178(56.3)\end{array}$ & $P$ \\
\hline Age at diagnosis, years, median (IQR) & $45.9(39.5-53.7)$ & $48.6(40.9-56.7)$ & 0.047 \\
\hline Age at diagnosis & & & 0.040 \\
\hline$\leq 50$ years & $91(65.9)$ & $97(54.5)$ & \\
\hline$>50$ years & $47(34.1)$ & $81(45.5)$ & \\
\hline $\mathrm{cT}$ & & & 0.54 \\
\hline $\mathrm{T} 1$ & $23(16.7)$ & $28(15.8)$ & \\
\hline $\mathrm{T} 2$ & $74(53.6)$ & $101(57.1)$ & \\
\hline $\mathrm{T} 3$ & $38(27.5)$ & $40(22.6)$ & \\
\hline $\mathrm{T} 4$ & $3(2.2)$ & $8(4.5)$ & \\
\hline $\mathrm{cN}$ & & & 0.19 \\
\hline Node negative & $50(36.2)$ & $52(29.2)$ & \\
\hline Node positive & $88(63.8)$ & $126(70.8)$ & \\
\hline $\mathrm{cM}$ & & & 0.19 \\
\hline M0 & $129(93.5)$ & $172(96.6)$ & \\
\hline M1 & $9(6.5)$ & $6(3.4)$ & \\
\hline Tumor size MRI before NST ${ }^{b}$ & & & 0.45 \\
\hline $0-35 \mathrm{~mm}$ & $73(54.1)$ & $88(49.2)$ & \\
\hline $36-120 \mathrm{~mm}$ & $62(45.9)$ & $89(50.3)$ & \\
\hline MRI size, mm, median (IQR) & $34(24-60)$ & $36(24-52)$ & 0.66 \\
\hline Suspicious calcifications $\mathrm{Mx}$ & & & 0.005 \\
\hline Absent & $41(29.9)$ & $79(45.4)$ & \\
\hline Present & $96(70.1)$ & $95(54.6)$ & \\
\hline Extent of suspicious calcifications ${ }^{b}$ & & & 0.031 \\
\hline $5-55 \mathrm{~mm}$ & $23(41.8)$ & $32(62.8)$ & \\
\hline $56-140 \mathrm{~mm}$ & $32(58.2)$ & $19(37.3)$ & \\
\hline Area suspicious calcifications, mm, median (IQR) & $60(35-88)$ & $50(20-70)$ & 0.022 \\
\hline IBC subtype & & & 0.003 \\
\hline No special type ${ }^{c}$ & $133(96.4)$ & $150(84.8)$ & \\
\hline Lobular & $2(1.5)$ & $15(8.5)$ & \\
\hline Other & $3(2.2)$ & $12(6.8)$ & \\
\hline Grade IBC $^{\mathrm{d}}$ & & & 0.054 \\
\hline Grade $1+2$ & $71(52.2)$ & $70(41.2)$ & \\
\hline Grade 3 & $65(47.8)$ & $100(58.8)$ & \\
\hline HR status IBC & & & 0.58 \\
\hline HR negative & $60(43.5)$ & $83(46.6)$ & \\
\hline HR positive & $78(56.5)$ & $95(53.4)$ & \\
\hline $\mathrm{Ki}-67 \mathrm{IBC}, \%$ & & & 0.45 \\
\hline Low, $\leq 20$ & $40(39.2)$ & $44(34.4)$ & \\
\hline High, $>20$ & $62(60.8)$ & $84(65.6)$ & \\
\hline Chemotherapy & & & 0.79 \\
\hline Taxanes & $111(80.4)$ & $147(82.6)$ & \\
\hline Anthracyclines + taxanes & $26(18.8)$ & $29(16.3)$ & \\
\hline Other & $1(0.7)$ & $2(1.1)$ & \\
\hline HER2-blockade & & & 0.37 \\
\hline Tzt & $84(60.9)$ & $117(65.7)$ & \\
\hline $\mathrm{Tzt}+\mathrm{Ptz}$ & $54(39.1)$ & $61(34.3)$ & \\
\hline Type of surgery & & & 0.11 \\
\hline Breast-conserving surgery & $73(52.9)$ & $110(61.8)$ & \\
\hline Mastectomy & $65(47.1)$ & $68(38.2)$ & \\
\hline Response on MRI & & & 0.096 \\
\hline
\end{tabular}


Table 1 (continued)

\begin{tabular}{lccc}
\hline Factors & $\begin{array}{l}\text { DCIS } n(\%)^{\mathrm{a}} \\
n=138(43.7)\end{array}$ & $\begin{array}{l}\text { No DCIS } n(\%) \\
n=178(56.3)\end{array}$ & $P$ \\
\hline No/partial response & $24(18.3)$ & $43(26.5)$ \\
(Near)complete response & $107(81.7)$ & $119(73.5)$ & \\
\hline
\end{tabular}

$I B C$ invasive breast cancer, $n$ number, $P P$ value, $I Q R$ interquartile range, NST neoadjuvant systemic therapy, $M x$ mammography, $H R$ hormone receptor, $T z t$ trastuzumab, $P t z$ pertuzumab

${ }^{a}$ One woman had bilateral breast cancer

${ }^{\mathrm{b}}$ Tumor size on MRI before NST and extent of suspicious calcifications on mammography were categorized into two groups with the median in this group of 316 patients used as cut-off value

${ }^{c}$ Formerly known as invasive ductal carcinoma

${ }^{\mathrm{d}}$ Grade IBC: only 1 patient had IBC grade 1 and did not have adjacent DCIS

Table 2 Pathologic findings after NST in patients with and without DCIS in pre-NST biopsy

\begin{tabular}{llllll}
\hline & \multicolumn{2}{l}{$\begin{array}{l}\text { DCIS in pre-NST biopsy } n(\%) \\
n=138(43.7)\end{array}$} & & \multicolumn{2}{l}{$\begin{array}{l}\text { No DCIS in pre-NST biopsy } n(\%) \\
n=178(56.3)\end{array}$} \\
\cline { 2 - 3 } \cline { 5 - 6 } & DCIS post-NST & No DCIS post-NST & & DCIS post-NST & No DCIS post-NST \\
\hline IBC post-NST & $35(47.3)$ & $9(14.1)$ & $38(62.3)$ & $38(32.5)$ \\
No IBC post-NST & $39(52.7)$ & $55(85.9)$ & & $23(37.7)$ & $79(67.5)$ \\
Total $n$ & 74 & 64 & 61 & 117 \\
\hline
\end{tabular}

$N S T$ neoadjuvant systemic therapy, $n$ number, $I B C$ invasive breast cancer of calcifications in DCIS on pre-NST biopsy (OR 3.19; $95 \%$ CI 1.34-7.60) and Ki-67 expression $>20 \%$ in DCIS (OR 2.74; 95\% CI 1.09-6.89). Grade and HR status of IBC or DCIS was not associated with DCIS response. The number of patients with HER2-negative DCIS was too small to allow an informative analysis on the association of HER2 status in DCIS with treatment response.

All abovementioned, eligible factors except Ki-67 expression $>20 \%$ in DCIS, were also independently associated with DCIS response in multivariable analysis (see Supplementary Table 2). After multiple imputation, Ki-67 expression $>20 \%$ in DCIS no longer reached the significance level set for entry into multivariable analysis.

\section{Discussion}

We have demonstrated that a part of the DCIS lesions adjacent to HER2-positive breast cancer can be eradicated after NST. To the best of our knowledge, this is the largest study that examined the response of DCIS, found adjacent to HER2-positive IBC, to NST containing HER2-blockade and the first study that assessed the association of clinicopathological and radiological factors with response. The response evaluation of adjacent DCIS is highly relevant, as NST containing HER2-blockade frequently results in pCR of HER2-positive IBC, but the presence of extensive, clinically detectable DCIS pre-NST often precludes performing BCS. Therefore, it would be most relevant to know in which patients adjacent DCIS will respond to NST to eventually increase the conversion rate of mastectomy to breast-conserving surgery. We have identified several factors associated with the response of DCIS to NST that can aid towards selection of a subgroup among HER2-positive breast cancer patients with extensive DCIS that could be treated by breastconserving surgery.

In this study, we analyzed 316 women with HER2-positive IBC of whom 138 (44\%) had adjacent DCIS in their pre-NST biopsies. Our incidence rate of DCIS was in the same range as reported by others who also evaluated the presence of adjacent DCIS in pre-NST biopsies, i.e., 37-46\% in HER2-positive IBC [14, 24, 25]. However, a higher incidence rate of adjacent DCIS is seen in studies assessing its presence in surgical specimens of patients undergoing upfront surgery, i.e., 57-72\% in HER2-positive IBC [13, $15,17]$. Our finding of residual DCIS after NST in 61 out of 178 patients (34\%) without adjacent DCIS in their pre-NST biopsies underlines that identifying patients with adjacent DCIS in biopsies, targeting the invasive component, is less accurate.

Studies have suggested that IBC with adjacent DCIS is associated with less aggressive behavior compared to IBC without DCIS with significantly better overall survival (5-year overall survival, $89 \%$ versus $86 \%, P<0.001$ ) [13, 15]. Compared to IBC without DCIS, IBC with adjacent DCIS was associated with a lower Ki-67 expression and grade, ER/PR/HER2 positivity, lower tumor and nodal stage, and was more frequently found in pre-menopausal women $[13,15]$. In our study, IBC with adjacent DCIS was associated with a younger age and the presence of suspicious 
Table 3 Associations of clinicoradiological and IBC factors with response ${ }^{\mathrm{a}}$ of DCIS to NST in univariable analysis

\begin{tabular}{|c|c|c|c|c|c|}
\hline Clinico-radiological factors & Total $n(\%)$ & $\begin{array}{l}\text { Response } n(\%) \\
n=64(46.4)\end{array}$ & $\begin{array}{l}\text { No response } n(\%) \\
n=74(53.6)\end{array}$ & $\mathrm{OR}^{\mathrm{b}}(95 \% \mathrm{CI})^{\mathrm{c}}$ & $P^{\mathrm{d}}$ \\
\hline \multicolumn{6}{|l|}{ Age at diagnosis } \\
\hline$\leq 50$ years & $91(65.9)$ & $37(57.8)$ & $54(73.0)$ & REF & \\
\hline$>50$ years & $47(34.1)$ & $27(42.2)$ & $20(27.0)$ & $1.97(0.97-4.02)$ & 0.061 \\
\hline \multicolumn{6}{|l|}{ Chemotherapy } \\
\hline Taxanes & $111(80.4)$ & $50(78.1)$ & $61(82.4)$ & REF & \\
\hline Anthracyclines + taxanes & $26(18.8)$ & $13(20.3)$ & 13 (17.6) & $1.22(0.52-2.87)$ & \\
\hline Other & $1(0.7)$ & $1(1.6)$ & & NA & 0.65 \\
\hline \multicolumn{6}{|l|}{ HER2-blockade } \\
\hline Tzt & $84(60.9)$ & $32(50.0)$ & $52(70.3)$ & REF & \\
\hline $\mathrm{Tzt}+\mathrm{Ptz}$ & $54(39.1)$ & $32(50.0)$ & $22(29.7)$ & $2.36(1.17-4.75)$ & 0.015 \\
\hline \multicolumn{6}{|l|}{ Tumor size MRI before $\mathrm{NST}^{\mathrm{e}}$} \\
\hline $7-34 \mathrm{~mm}$ & $69(50.0)$ & $34(53.1)$ & $35(47.3)$ & $1.24(0.63-2.44)$ & 0.53 \\
\hline $35-110 \mathrm{~mm}$ & $66(47.8)$ & $29(45.3)$ & $37(50.0)$ & REF & \\
\hline Unknown & $3(2.2)$ & $1(1.6)$ & $2(2.7)$ & & \\
\hline \multicolumn{6}{|l|}{ Suspicious calcifications Mx } \\
\hline Absent & $41(29.7)$ & $28(43.8)$ & 13 (17.6) & $3.75(1.72-8.17)$ & \\
\hline Present & $96(69.6)$ & $35(54.7)$ & $61(82.4)$ & REF & 0.001 \\
\hline Unknown & $1(0.7)$ & $1(1.6)$ & & & \\
\hline \multicolumn{6}{|c|}{ Extent of suspicious calcifications $\mathrm{e}^{\mathrm{e}}$} \\
\hline $13-60 \mathrm{~mm}$ & $28(29.2)$ & $10(28.6)$ & $18(29.5)$ & REF & \\
\hline $61-140 \mathrm{~mm}$ & $27(28.1)$ & $11(31.4)$ & $16(26.2)$ & $1.24(0.42-3.68)$ & 0.70 \\
\hline Unknown & $41(42.7)$ & $14(40.0)$ & $27(44.3)$ & & \\
\hline \multicolumn{6}{|l|}{ Response on MRI } \\
\hline No/partial response & $24(17.4)$ & $6(9.4)$ & $18(24.3)$ & REF & \\
\hline (Near)complete response & $107(77.5)$ & $58(90.6)$ & $49(66.2)$ & $3.55(1.31-9.64)$ & 0.008 \\
\hline Unknown & $7(5.1)$ & & $7(9.5)$ & & \\
\hline \multicolumn{6}{|l|}{ IBC factors } \\
\hline \multicolumn{6}{|l|}{ Grade } \\
\hline Grade $1+2$ & $71(51.5)$ & $37(57.8)$ & $34(46.0)$ & $1.63(0.83-3.22)$ & \\
\hline Grade 3 & $65(47.1)$ & $26(40.6)$ & $39(52.7)$ & REF & 0.16 \\
\hline Unknown & $2(1.5)$ & $1(1.6)$ & $1(1.4)$ & & \\
\hline \multicolumn{6}{|l|}{ HR status } \\
\hline HR negative & $60(43.5)$ & $32(50.0)$ & $28(37.8)$ & $1.64(0.83-3.24)$ & 0.15 \\
\hline HR positive & $78(56.5)$ & $32(50.0)$ & $46(62.2)$ & REF & \\
\hline \multicolumn{6}{|l|}{$\mathrm{Ki}-67, \%$} \\
\hline Low, $\leq 20$ & $40(29.0)$ & $18(28.1)$ & $22(29.7)$ & REF & \\
\hline High, $>20$ & $62(44.9)$ & $30(46.9)$ & $32(43.2)$ & $1.15(0.52-2.54)$ & 0.74 \\
\hline Unknown & $36(26.1)$ & $16(25.0)$ & $20(27.0)$ & & \\
\hline
\end{tabular}

$I B C$ invasive breast cancer, NST neoadjuvant systemic therapy, $n$ number, $O R$ odds ratio, $C I$ confidence interval, $P P$ value, $R E F$ reference, $N A$ not applicable, $T z t$ trastuzumab, $P t z$ pertuzumab, $M x$ mammography, $H R$ hormone receptor

${ }^{a}$ Response is defined as complete eradication of DCIS after neoadjuvant systemic therapy

${ }^{b}$ Missings were not taken into account as a separate category

${ }^{\mathrm{c}}$ Confidence interval is Wald-based

${ }^{\mathrm{d}} P$ value is based on the LR-based test statistic

${ }^{\mathrm{e}}$ Tumor size on MRI before NST and extent of suspicious calcifications on mammography were categorized into two groups with the median used as cut-off value 
Table 4 Associations of DCIS factors with response ${ }^{\mathrm{a}}$ of DCIS to NST in univariable analysis

\begin{tabular}{|c|c|c|c|c|c|}
\hline DCIS factors & $\begin{array}{l}\text { Total } n(\%) \\
n=138\end{array}$ & $\begin{array}{l}\text { Response } n(\%) \\
n=64(46.4)\end{array}$ & $\begin{array}{l}\text { No response } n(\%) \\
n=74(53.6)\end{array}$ & $\mathrm{OR}^{\mathrm{b}}(95 \% \mathrm{CI})^{\mathrm{c}}$ & $P^{\mathrm{d}}$ \\
\hline \multicolumn{6}{|l|}{ Grade $^{e}$} \\
\hline Grade $1+2$ & $63(45.7)$ & $27(42.2)$ & $36(48.7)$ & REF & \\
\hline Grade 3 & $72(52.2)$ & $37(57.8)$ & $35(47.3)$ & $1.41(0.71-2.78)$ & 0.32 \\
\hline Unknown & $3(2.2)$ & & $3(4.1)$ & & \\
\hline \multicolumn{6}{|l|}{ Growth pattern ${ }^{\mathrm{f}}$} \\
\hline (Non)solid & $22(15.9)$ & $8(12.5)$ & 14 (18.9) & REF & \\
\hline Solid & $110(79.7)$ & $54(84.4)$ & $56(75.7)$ & $1.69(0.66-4.34)$ & 0.27 \\
\hline Unknown & $6(4.4)$ & $2(3.1)$ & $4(5.4)$ & & \\
\hline \multicolumn{6}{|l|}{ Calcifications } \\
\hline Absent & $99(71.7)$ & $55(85.9)$ & $44(59.5)$ & $3.19(1.34-7.60)$ & 0.006 \\
\hline Present & $32(23.2)$ & $9(14.1)$ & $23(31.1)$ & REF & \\
\hline Unknown & $7(5.1)$ & & $7(9.5)$ & & \\
\hline \multicolumn{6}{|l|}{ Necrosis } \\
\hline Absent & $69(50.0)$ & $39(60.9)$ & $30(40.5)$ & $1.98(0.99-3.95)$ & 0.053 \\
\hline Present & $63(45.7)$ & $25(39.1)$ & $38(51.4)$ & REF & \\
\hline Unknown & $6(4.4)$ & & $6(8.1)$ & & \\
\hline \multicolumn{6}{|l|}{ Mitoses } \\
\hline Sparse & $82(59.4)$ & $38(59.4)$ & $44(59.5)$ & REF & \\
\hline Many & $48(34.8)$ & $23(35.9)$ & $25(33.8)$ & $1.07(0.52-2.17)$ & 0.86 \\
\hline Unknown & $8(5.8)$ & $3(4.7)$ & $5(6.8)$ & & \\
\hline \multicolumn{6}{|l|}{ Periductal fibrosis } \\
\hline Absent + subtle & $71(51.5)$ & $32(50.0)$ & $39(52.7)$ & REF & \\
\hline Prominent & $53(38.4)$ & $27(42.2)$ & $26(35.1)$ & $1.27(0.62-2.58)$ & 0.52 \\
\hline Unknown & $14(10.1)$ & $5(7.8)$ & $9(12.2)$ & & \\
\hline \multicolumn{6}{|l|}{ Type fibrosis ${ }^{\mathrm{g}}$} \\
\hline Sclerotic & $41(46.1)$ & $17(42.5)$ & $24(49.0)$ & REF & \\
\hline Myxoid & $47(52.8)$ & $23(57.5)$ & $24(49.0)$ & $1.35(0.58-3.15)$ & 0.48 \\
\hline Unknown & $1(1.1)$ & & $1(2.0)$ & & \\
\hline \multicolumn{6}{|c|}{ Lymphocytic infiltrate } \\
\hline Absent + subtle & $99(71.7)$ & $45(70.3)$ & $54(73.0)$ & REF & \\
\hline Prominent & $27(19.6)$ & $14(21.9)$ & $13(17.6)$ & $1.29(0.55-3.03)$ & 0.56 \\
\hline Unknown & $12(8.7)$ & $5(7.8)$ & $7(9.5)$ & & \\
\hline \multicolumn{6}{|l|}{ HR status } \\
\hline HR negative & $31(22.5)$ & $15(23.4)$ & $16(21.6)$ & $1.17(0.48-2.84)$ & 0.73 \\
\hline HR positive & $54(39.1)$ & $24(37.5)$ & $30(40.5)$ & REF & \\
\hline Unknown & $53(38.4)$ & $25(39.1)$ & $28(37.8)$ & & \\
\hline \multicolumn{6}{|l|}{$\mathrm{Ki}-67, \%$} \\
\hline Low, $\leq 20$ & $39(28.3)$ & $14(21.9)$ & $25(33.8)$ & REF & \\
\hline High, $>20$ & $38(27.5)$ & $23(35.9)$ & $15(20.3)$ & $2.74(1.09-6.89)$ & 0.030 \\
\hline Unknown & $61(44.2)$ & $27(42.2)$ & $34(46.0)$ & & \\
\hline
\end{tabular}

$N S T$ neoadjuvant systemic therapy, $n$ number, $O R$ odds ratio, $C I$ confidence interval, $P P$ value, $R E F$ reference, $H R$ hormone receptor

${ }^{a}$ Response is defined as complete eradication of DCIS after neoadjuvant systemic therapy

${ }^{b}$ Missings were not taken into account as a separate category

${ }^{\mathrm{c}}$ Confidence interval is Wald-based

${ }^{\mathrm{d}} P$ value is based on the LR-based test statistic

${ }^{\mathrm{e}}$ Grade DCIS: only 2 patients had grade 1

f(Non)solid = clinging, (micro)papillary, cribriform

${ }^{\mathrm{g}}$ Type of fibrosis was only scored when periductal fibrosis was present 
calcifications on pre-NST mammography. In addition, DCIS was more often found adjacent to IBC grade $1+2$, but this association did not reach statistical significance. Two other studies that evaluated the sensitivity of DCIS to NST did not find a correlation between the presence of adjacent DCIS and age, nodal status, IBC grade, HR status or Ki-67 [14, 24]. As these studies, like ours, were performed in women treated by NST partly focusing on HER2-positive IBC alone, and likely suboptimally identifying IBC with adjacent DCIS in pre-NST biopsies, associations may be different.

We found that DCIS was eradicated after NST in 64 out of 138 women with adjacent DCIS in their pre-NST biopsies $(46 \%)$. Our results are in line with those of a smaller study by von Minckwitz et al., in which DCIS was eradicated in $30 / 59$ patients $(51 \%)$ with HER2-positive IBC who were treated with a neoadjuvant regimen including anthracyclines, taxanes and trastuzumab with or without capecitabine [24]. A slightly lower, but still comparable response rate of $36 \%$ was found in a study, which also focused on adjacent DCIS in HER2-positive IBC, in which patients were treated with taxane-based chemotherapy plus trastuzumab and also pertuzumab in a small subgroup [25]. Another study showed a pCR of DCIS, found adjacent to IBC of all subtypes, in 10 out of 30 patients (33\%) treated with anthracycline-taxanecontaining NST (plus trastuzumab when the HER2 receptor was overexpressed) [14].

Absence of suspicious calcifications on pre-NST mammography, dual HER2-blockade, a (near) complete response on MRI, the absence of calcifications in DCIS on pre-NST biopsy and a Ki-67 expression in DCIS of $>20 \%$ were associated with response of DCIS to NST in univariable analysis. The results for Ki-67 expression in DCIS should be interpreted with some caution due to the large proportion of missings. Reports on response of invasive HER2positive breast cancer have identified similar factors, as complete response is more frequently observed in patients treated with dual HER2-blockade compared to trastuzumab alone, in patients with a (near) complete response on MRI or in IBC with a high Ki-67 expression [4-6, 8, 34, 35]. A recent review concerning HER2-positive IBC showed that three factors are associated with an increased pCR rate: (1) high HER2 combined with low estrogen receptor 1 gene expression levels, (2) a 'HER2-enriched' PAM50 intrinsic subtype, and (3) higher levels of tumor infiltrating lymphocytes [36]. Although we did not perform gene expression analysis, we evaluated HR status of IBC and DCIS, but did not find a higher response rate for HR-negative IBC or DCIS. It could be that response rates of HR-negative versus HR-positive DCIS does not parallel the situation for IBC in HER2-positive breast cancer patients. In our study cohort, women with HR-negative IBC did not differ from women with HR-positive IBC in terms of age, treatment, and grade or proliferation of IBC. There was a trend towards smaller tumor size in HR-positive IBC compared to HR-negative IBC based on T-stage and MRI size at baseline $(P=0.065$ and $P=0.074$, respectively), but this does not imply an association with a smaller size of the DCIS component per se. Perhaps a discordancy in HR status between DCIS and IBC may play a role here, but this seems unlikely when considering the small subset of such patients found in our cohort, of whom HR-negative IBC patients with adjacent HR-positive DCIS showed a higher response rate than HR-positive IBC patients with adjacent HR-negative DCIS (response rate $67 \%$ versus $50 \%$ ). Lastly, HR status of DCIS was missing for $38 \%$ of all cases included that could mask an underlying difference in response rates between HR-positive versus HR-negative DCIS.

Our study has several limitations. One limitation is a lack of thorough radiological correlation with DCIS prior to NST, which would enable more accurate identification of patients with (extensive) DCIS, allowing for more accurate assessment of true response. A second limitation is intrinsic to the way in which IBC is diagnosed and classified prior to NST, i.e., by taking a biopsy targeted on the IBC and pathologic evaluation thereof. This implies that the aim of most biopsies is not to assess the presence of adjacent DCIS. This may compromise adequate evaluation of the response of DCIS to NST, as there is a risk of missing adjacent DCIS in pre-NST biopsies. Expanding our analysis by including patients who only showed (residual) DCIS after NST would enable rightful recognition of these 'non-complete responders'. However, this would also lead to an underestimation of DCIS response because patients without DCIS in pre-NST biopsies who had a complete response would not be considered. In this context it is also important to note that in clinical practice DCIS can be occult on imaging, representing a subset of patients in whom adjacent DCIS was only identified after NST in our study. For these patients, prediction of DCIS response will not change surgical treatment decisions. A third potential minor limitation might be that the diagnostic biopsy procedure results in complete removal of a small component of adjacent DCIS, compromising response evaluation. Yet, as feasibility issues for breast-conserving surgery particularly arise in patients with extensive adjacent DCIS, it is unlikely that this will impact clinical practice.

In conclusion, we demonstrated in this exploratory study that complete response of DCIS to NST can be achieved in almost half of the patients with confirmed DCIS adjacent to HER2-positive IBC in pre-NST biopsies. Further research is needed to validate our findings within HER2-positive breast cancer patients with clinically detectable, extensive DCIS, while carefully correlating radiology and pathology of the DCIS component pre- and post-NST. Within such a context, the conversion rate of mastectomy to breast-conserving surgery, and recurrence and survival rates related to DCIS response could be evaluated. For now, our study indicates 
that the presence of extensive DCIS in HER2-positive breast cancer before NST should not always indicate a mastectomy, and the predictive factors we found could be helpful when considering BCS in these patients.

Supplementary Information The online version contains supplementary material available at https://doi.org/10.1007/s10549-021-06235-2.

Acknowledgements The authors like to acknowledge the NKI-AVL Core Facility Molecular Pathology \& Biobanking (CFMPB) for supplying lab support. This work was supported by Cancer Research UK and by KWF Kankerbestrijding (Ref. C38317/A24043).

Author contributions EJG, MEMvdN, MSvR, MS, FvD, and JW were responsible for the study design. EJG and MEMvdN coordinated the study. EJG analyzed the data under supervision of MS. EJG and MEM$\mathrm{vdN}$ wrote the manuscript with significant contributions by all authors. All authors read and approved the final manuscript.

Funding This work was supported by Cancer Research UK and by KWF Kankerbestrijding (Ref. C38317/A24043).

Data availability The datasets generated during and/or analyzed during the current study are available from the corresponding author upon reasonable request. Requests should be made to Prof. Jelle Wesseling (j.wesseling@nki.nl).

\section{Declarations}

Conflict of interest Dr Sonke reports receiving institutional research support from AstraZeneca, Merck, Novartis, and Roche and Dr Mann from Siemens Healthineers, Medtronic, Bayer Healthcare, BD, Screenpoint Medical, Seno Medical and Transonic Imaging. For the remaining authors, none were declared.

Ethical approval This retrospective study involving human participants was in accordance with the ethical standards of the institutional and/or national research committee and with the 1964 Helsinki declaration and its later amendments or comparable ethical standards. This study was approved by the institutional review board of the NKI-AVL.

Consent to participate The study used only unidentifiable patient information, and no informed consent was required.

Consent to publish Not applicable.

Open Access This article is licensed under a Creative Commons Attribution 4.0 International License, which permits use, sharing, adaptation, distribution and reproduction in any medium or format, as long as you give appropriate credit to the original author(s) and the source, provide a link to the Creative Commons licence, and indicate if changes were made. The images or other third party material in this article are included in the article's Creative Commons licence, unless indicated otherwise in a credit line to the material. If material is not included in the article's Creative Commons licence and your intended use is not permitted by statutory regulation or exceeds the permitted use, you will need to obtain permission directly from the copyright holder. To view a copy of this licence, visit http://creativecommons.org/licenses/by/4.0/.

\section{References}

1. Buzdar AU, Ibrahim NK, Francis D, Booser DJ, Thomas ES, Theriault RL et al (2005) Significantly higher pathologic complete remission rate after neoadjuvant therapy with trastuzumab, paclitaxel, and epirubicin chemotherapy: results of a randomized trial in human epidermal growth factor receptor 2-positive operable breast cancer. J Clin Oncol 23(16):3676-3685

2. Buzdar AU, Valero V, Ibrahim NK, Francis D, Broglio KR, Theriault RL et al (2007) Neoadjuvant therapy with paclitaxel followed by 5-fluorouracil, epirubicin, and cyclophosphamide chemotherapy and concurrent trastuzumab in human epidermal growth factor receptor 2-positive operable breast cancer: an update of the initial randomized study. Clin Cancer Res 13(1):228-233

3. Gianni L, Eiermann W, Semiglazov V, Manikhas A, Lluch A, Tjulandin S et al (2010) Neoadjuvant chemotherapy with trastuzumab followed by adjuvant trastuzumab versus neoadjuvant chemotherapy alone, in patients with HER2-positive locally advanced breast cancer (the NOAH trial): a randomised controlled superiority trial with a parallel HER. Lancet 375(9712):377-384. https://doi.org/10.1016/S0140-6736(09) 61964-4

4. Chen S, Liang Y, Feng Z, Wang M (2019) Efficacy and safety of HER2 inhibitors in combination with or without pertuzumab for HER2-positive breast cancer: a systematic review and metaanalysis. BMC Cancer 19(1):1-15

5. Urruticoechea A, Rizwanullah M, Im SA, Sánchez Ruiz AC, Láng I, Tomasello G et al (2017) Randomized phase III trial of trastuzumab plus capecitabine with or without pertuzumab in patients with human epidermal growth factor receptor 2-positive metastatic breast cancer who experienced disease progression during or after trastuzumab-based therap. J Clin Oncol 35(26):3030-3038

6. Gianni L, Pienkowski T, Im YH, Tseng LM, Liu MC, Lluch A et al (2016) 5-year analysis of neoadjuvant pertuzumab and trastuzumab in patients with locally advanced, inflammatory, or earlystage HER2-positive breast cancer (NeoSphere): a multicentre, open-label, phase 2 randomised trial. Lancet Oncol 17(6):791-800

7. Schneeweiss A, Chia S, Hickish T, Harvey V, Eniu A, Hegg R et al (2013) Pertuzumab plus trastuzumab in combination with standard neoadjuvant anthracycline-containing and anthracyclinefree chemotherapy regimens in patients with HER2-positive early breast cancer: a randomized phase II cardiac safety study (TRYPHAENA). Ann Oncol 24(9):2278-2284. https://doi.org/10.1093/ annonc/mdt 182

8. Beitsch P, Whitworth P, Baron P, Rotkis MC, Mislowsky AM, Richards PD et al (2017) Pertuzumab/trastuzumab/CT versus trastuzumab/CT therapy for HER2+ breast cancer: results from the prospective neoadjuvant breast registry symphony trial (NBRST). Ann Surg Oncol 24(9):2539-2546

9. van Ramshorst MS, van der Voort A, van Werkhoven ED, Mandjes IA, Kemper I, Dezentjé VO et al (2018) Neoadjuvant chemotherapy with or without anthracyclines in the presence of dual HER2 blockade for HER2-positive breast cancer (TRAIN-2): a multicentre, open-label, randomised, phase 3 trial. Lancet Oncol 19(12):1630-1640

10. Shin HC, Han W, Moon HG, Im SA, Moon WK, Park IA et al (2013) Breast-conserving surgery after tumor downstaging by neoadjuvant chemotherapy is oncologically safe for stage III breast cancer patients. Ann Surg Oncol 20(8):2582-2589 
11. Mieog JS, Van Der Hage J, van de Velde CJH (2007) Preoperative chemotherapy for women with operable breast cancer (Review ). Cochrane Database Syst Rev 18(2):1-63

12. Cain H, Macpherson IR, Beresford M, Pinder SE, Pong J, Dixon JM (2017) Neoadjuvant therapy in early breast cancer: treatment considerations and common debates in practice. Clin Oncol 29(10):642-652. https://doi.org/10.1016/j.clon.2017.06.003

13. Wong H, Lau S, Yau T, Cheung P, Epstein RJ (2010) Presence of an in situ component is associated with reduced biological aggressiveness of size-matched invasive breast cancer. Br J Cancer 102(9):1391-1396. https://doi.org/10.1038/sj.bjc.6605655

14. Goldberg H, Zandbank J, Kent V, Leonov-Polak M, Livoff A, Chernihovsky A et al (2017) Chemotherapy may eradicate ductal carcinoma in situ (DCIS) but not the associated microcalcifications. Eur J Surg Oncol 43(8):1415-1420. https://doi.org/10. 1016/j.ejso.2017.04.011

15. Kole AJ, Park HS, Johnson SB, Kelly JR, Moran MS, Patel AA (2019) Overall survival is improved when DCIS accompanies invasive breast cancer. Sci Rep 9(1):1-9. https://doi.org/10.1038/ s41598-019-46309-2

16. Li JJ, Chen C, Gu Y, Di G, Wu J, Liu G et al (2014) The role of mammographic calcification in the neoadjuvant therapy of breast cancer imaging evaluation. PLoS One 9(2):17-19

17. Doebar SC, van den Broek EC, Koppert LB, Jager A, Baaijens MHA, Obdeijn IMAM et al (2016) Extent of ductal carcinoma in situ according to breast cancer subtypes: a population-based cohort study. Breast Cancer Res Treat 158(1):179-187

18. Wu W, Kamma H, Ueno E, Fujiwara M, Satoh H, Hara $\mathrm{H}$ et al (2002) The intraductal component of breast cancer is poorly responsive to neo-adjuvant chemotherapy. Oncol Rep 9(5):1027-1031

19. Mazouni C, Peintinger F, Wan-Kau S, Andre F, Gonzalez-Angulo AM, Symmans WF et al (2007) Residual ductal carcinoma in situ in patients with complete eradication of invasive breast cancer after neoadjuvant chemotherapy does not adversely affect patient outcome. J Clin Oncol 25(19):2650-2655

20. Pusztai L, Siddik ZH, Mills GB, Bast RC (1998) Physiologic and pathologic drug resistance in ovarian carcinoma-a hypothesis based on a clonal progression model. Acta Oncol (Madr) 37(7-8):629-640

21. Holmes D, Colfry A, Czerniecki B, Dickson-Witmer D, Francisco Espinel C, Feldman E et al (2015) Performance and practice guideline for the use of neoadjuvant systemic therapy in the management of breast cancer. Ann Surg Oncol 22(10):3184-3190

22. Netherlands Comprehensive Cancer Organization (IKNL) Breast cancer Dutch guideline, version 2.0 authorized 2020. Methodology: evidence based, accountability: NABON. https://www.oncol ine.nl/borstkanker

23. Matsuo K, Fukutomi T, Watanabe T, Hasegawa T, Tsuda H, Akashi-Tanaka S (2002) Concordance in pathological response to neoadjuvant chemotherapy between invasive and noninvasive components of primary breast carcinomas. Breast Cancer $9(1): 75-81$

24. Von Minckwitz G, Darb-Esfahani S, Loibl S, Huober J, Tesch $\mathrm{H}$, Solbach C et al (2012) Responsiveness of adjacent ductal carcinoma in situ and changes in HER2 status after neoadjuvant chemotherapy/trastuzumab treatment in early breast cancerresults from the GeparQuattro study (GBG 40). Breast Cancer Res Treat 132(3):863-870

25. Sun $S$, van la Parra RFD, Rauch GM, Checka C, Tadros AB, Lucci A et al (2019) Patient selection for clinical trials eliminating surgery for HER2-positive breast cancer treated with neoadjuvant systemic therapy. Ann Surg Oncol 26(10):3071-3079. https://doi. org/10.1245/s10434-019-07533-2

26. Adrada BE, Huo L, Lane DL, Arribas EM, Resetkova E, Yang W (2015) Histopathologic correlation of residual mammographic microcalcifications after neoadjuvant chemotherapy for locally advanced breast cancer. Ann Surg Oncol 22(4):1111-1117

27. Weiss A, Lee KC, Romero Y, Ward E, Kim Y, Ojeda-Fournier $\mathrm{H}$ et al (2014) Calcifications on mammogram do not correlate with tumor size after neoadjuvant chemotherapy. Ann Surg Oncol 21(10):3310-3316

28. Wolff AC, Hammond MEH, Hicks DG, Dowsett M, McShane LM, Allison KH et al (2013) Recommendations for human epidermal growth factor receptor 2 testing in breast. J Clin Oncol 31(31):3997-4013

29. D'Orsi C, Sickles E, Mendelson E, Morris E et al (2013) ACR BI-RADS ${ }^{\circledR}$ Atlas, breast imaging reporting and data system. American College of Radiology, Reston

30. Marinovich ML, Macaskill P, Irwig L, Sardanelli F, Mamounas E, von Minckwitz G et al (2015) Agreement between MRI and pathologic breast tumor size after neoadjuvant chemotherapy, and comparison with alternative tests: individual patient data meta-analysis. BMC Cancer 15(1):1-12. https://doi.org/10.1186/ s12885-015-1664-4

31. Janssen NNY, Nijkamp J, Alderliesten T, Loo CE, Rutgers EJT, Sonke JJ et al (2016) Radioactive seed localization in breast cancer treatment. Br J Surg 103(1):70-80

32. Holland R, Peterse JL, Millis RR, Eusebi V, Faverly D, Van de Vijver MJ et al (1994) Ductal carcinoma in situ: a proposal for a new classification. Semin Diagn Pathol 11(3):167-180

33. Rubin DB (1987) Multiple imputation for nonresponse in surveys. Wiley, New York

34. Swain SM, Baselga J, Kim SB, Ro J, Semiglazov V, Campone M et al (2014) Pertuzumab, trastuzumab, and docetaxel in HER2positive metastatic breast cancer. N Engl J Med 372(8):724-734

35. Tao M, Chen S, Zhang X, Zhou Q (2017) Ki-67 labeling index is a predictive marker for a pathological complete response to neoadjuvant chemotherapy in breast cancer: a meta-analysis. Medicine (United States) 96(51):e9384

36. Brandão M, Caparica R, Malorni L, Prat A, Carey LA, Piccart $M$ (2020) What is the real impact of estrogen receptor status on the prognosis and treatment of HER2-positive early breast cancer? Clin Cancer Res. https://doi.org/10.1158/1078-0432. CCR-19-2612

Publisher's Note Springer Nature remains neutral with regard to jurisdictional claims in published maps and institutional affiliations. 


\section{Authors and Affiliations}

\section{Emma J. Groen ${ }^{1,2} \cdot$ Marieke E. M. van der Noordaa $^{3} \cdot$ Michael Schaapveld $^{4} \cdot$ Gabe S. Sonke $^{5}$ Ritse M. Mann ${ }^{6,7}$.} Mette S. van Ramshorst ${ }^{8}$ - Esther H. Lips ${ }^{2}$ - Marie-Jeanne T. F. D. Vrancken Peeters ${ }^{3}$. Frederieke H. van Duijnhoven ${ }^{3}$. Jelle Wesseling ${ }^{1,2,9}$

1 Department of Pathology, Netherlands Cancer Institute - Antoni van Leeuwenhoek, Plesmanlaan 121, 1066 CX Amsterdam, The Netherlands

2 Division of Molecular Pathology, Netherlands Cancer Institute - Antoni van Leeuwenhoek, Amsterdam, The Netherlands

3 Department of Surgical Oncology, Netherlands Cancer Institute - Antoni van Leeuwenhoek, Amsterdam, The Netherlands

4 Division of Psychosocial Research and Epidemiology, Netherlands Cancer Institute - Antoni van Leeuwenhoek, Amsterdam, The Netherlands
5 Department of Medical Oncology, Netherlands Cancer Institute - Antoni van Leeuwenhoek, Amsterdam, The Netherlands

6 Department of Radiology, Netherlands Cancer Institute Antoni van Leeuwenhoek, Amsterdam, The Netherlands

7 Department of Radiology and Nuclear Medicine, Radboud University Medical Center, Nijmegen, The Netherlands

8 Department of Internal Medicine, Onze Lieve Vrouwe Gasthuis, Amsterdam, The Netherlands

9 Department of Pathology, Leiden University Medical Center, Leiden, The Netherlands 